\title{
В.М. Маракулин
}

Новосибирский государственный университет;

Институт математики им. С.Л. Соболева СО РАН, Новосибирск

\section{О существовании пространственного равновесия и обобщенных теоремах о неподвижной точке}

\begin{abstract}
Аннотация. Изучается задача иммиграционно-состоятельного (стабильного) деления конечномерной области на юрисдикции (страны). Это своеобразное равновесие Тибу, где принцип миграционной состоятельности предполагает, что у жителей отсутствуют выявленные мотивы для изменения юрисдикции. В частности, у граждан граничных стран в данной точке размещения населения имеется равенство издержек. Предполагается, что задана измеримая плотность распределения населения, и требуется, чтобы межстрановая граница была представлена непрерывной поверхностью (кривой). Доказательство существования требуемого деления основано на сведении проблемы к нахождению неподвижной точки. Однако при этом нарушаются условия классических теорем БрауэраКакутани или конической теоремы Красносельского. Это привело к разработке их обобщения на случай отображения, возможно, действующего за пределы компакта. Доказано, что непрерывное отображение, определенное на выпуклом компакте, обладает неподвижной точкой, если удовлетворяет одному из двух граничных условий: сжатие или расширение. Этот результат распространяется и на точечно-множественные отображения Какутани. Применяя его, мы доказываем существование иммиграционно состоятельного деления на страны любой компактной конечномерной области в достаточно общей постановке.
\end{abstract}

Ключевые слова: теоремы о неподвижной точке Брауәра и Какутани, сжимаюоее и расширяющее условия, странообразование, миграчионно состоятельное (стабильное) разбиение.

Классификация JEL: D70, H20, H73.

DOI: $10.31737 / 2221-2264-2019-42-2-1$

\section{Введение}

В основополагающей работе (Alesina, Spolaore, 1997) была предложена базовая модель странообразования, в которой издержки населения описываются как сумма двух величин - отношения совокупных издержек на общую массу населения страны и транспортные издержки до центра государства. Эта модель изучалась в ряде последующих работ, однако в каждой из них рассматривается случай одномерной области и стран интервального вида (странообразование на отрезке $[0,1])$. Первые результаты в части разрешения проблемы существования были получены в (Musatov et al., 2016), где с применением леммы Гейла-Никайдо-Дебре было доказано существование иммиграционно состоятельного разбиения отрезка на страны-интервалы, т.е. такого, в котором отсутствуют стимулы к изменению страны проживания. В (Маракулин, 2017) математическая часть этого подхода была существенно усилена и распространена на случай распределения населения, описанного как сумма дискретной меры и меры абсо- 
лютно непрерывной относительно меры Лебега (имеет измеримую плотность). Дискретная мера моделирует городское население, а абсолютно непрерывная - сельское.

Первое значимое решение, распространяющее эти результаты (теорему существования) на случай 2-мерной (и более) области, появляется в (Savvateev et al., 2018). Представленное там доказательство весьма элегантно и основано на применении ККМ-леммы (Кнастера, Куратовского, Мазуркевича). В первоначальной версии этой работы достигнутый результат был ограничен наличием фиксированных в пространстве столиц; однако в последнем варианте была предложена существенно более общая модель и доказано существование нетривиального равновесного деления на страны. В (Marakulin, 2016, 2017) условия, обеспечивающие существование миграционно-состоятельного деления на страны, были существенно ослаблены при следующих допущениях: переменное положение центра страны, возможное наличие в пространстве незаселенных зон и т.д., однако эти результаты все еще недостаточно общие (подробности см. ниже) и требуют дальнейшей разработки.

Проведенный в (Savvateev et al., 2018) анализ показал, что проблему существования стабильного деления на страны (непрерывные границы, заданное число стран, у граждан которых отсутствует стимул менять юрисдикцию) можно свести к вопросу о существовании неподвижной точки у непрерывного отображения, принимающего значения в стандартном симплексе $\Delta^{(n-1)}=\left\{\left(\delta_{1}, \ldots, \delta_{n}\right) \in \mathbb{R}_{+}^{n} \mid \sum \delta_{i}=1\right\}$, но заданного на его $\varepsilon$-сжатии $\Delta_{\varepsilon}^{(n-1)}=\left\{\delta \in \Delta^{(n-1)} \mid \delta_{i} \geq \varepsilon \quad \forall i=1, \ldots, n\right\}, \quad \varepsilon>0$. Требуется найти неподвижную точку в меньшем симплексе. Эта точка существует в силу ключевого свойства отображения, состоящего в том, что грани ع-симплекса отображаются в соответствующие грани стандартного, но такие теоремы ранее не были мне известны. Соответствующий результат был получен как следствие новой теоремы о неподвижной точке, в которой требование принимать значение в области определения $X \subset \mathbb{R}^{n}$ отображения $f$ (требуется $f(X) \subseteq X, X-$ выпуклый компакт) заменяется на слабое граничное условие. Это так называемые сжимающее и расширяющее свойства (названы по аналогии с конической теоремой Красносельского), которые применимы в случае $f(X) \nsubseteq X$. Например, расширяющее условие означает, что для каждого граничного $x \in \partial X$ и каждого линейного $h \neq 0$, опорного к $X$ в точке $x$, т.е. если $\langle h, X\rangle \leq\langle h, x\rangle$, имеет место $\langle h, f(x)\rangle \geq\langle h, x\rangle$. Изменяя знак последнего неравенства, мы приходим к сжимающему предположению. Утверждается, что в таком случае непрерывное отображение, заданное на $X$, имеет неподвижную точку.

Классическая теорема Брауэра утверждает, что в конечномерном пространстве любое непрерывное отображение выпуклого компакта в себя имеет неподвижную точку. Это очень удобная теорема, которая находит свои приложения в множестве задач теоретического 
и прикладного анализа. В течение XX в. условия этой теоремы многократно ослаблялись и приспосабливались к решению специфических прикладных задач ${ }^{1}$. Ослаблялись также требования к самому отображению, которое теперь уже может действовать и за пределы компакта, но при этом удовлетворяет определенным граничным условиям. Наиболее известное из них так называемое условие Лере-Шаудера, которое (упрощенно) формулируется следующим образом.

Пусть $X \subset \mathbb{R}^{n}$ конечномерный выпуклый компакт и $0 \in \operatorname{int} X$. Условие на отображение $f: X \rightarrow \mathbb{R}^{n}$ означает, что для каждого граничного $x \in X$ не существует действительного $\lambda>1$ такого, что $f(x)=\lambda x$. Альтернатива (теорема) Лере-Шаудера утверждает, что тогда непрерывное отображение имеет неподвижную точку на $X$ (см., например, (Dugundji, Granas, 1982, p. 61, Theorem 5.1)). Однако существуют приложения, в которых результатов типа Лере-Шаудера недостаточно. Теоремы о неподвижных точках, основанные на альтернативных граничных требованиях, появляются в работах Красносельского.

В современной литературе по неподвижным точкам известны два типа теорем, носящих имя Красносельского. В интересующем нас случае такая теорема появляется в (Красносельский, 1960), где рассматриваются непрерывные операторы, определенные и принимающие значения на выпуклом замкнутом заостренном конусе в банаховом пространстве (подробнее см. (Kwong, 2008)). Теорема утверждает, что в коническом отрезке существует неподвижная точка, если на границе отрезка выполнены условия сжатия или расширения. Теоремы этого типа (названные коническими) находятся в общем ряду значимых классических теорем о неподвижной точке, они распространяют классический подход на отображения, не обязательно переводящие область определения в себя, причем условие Лере-Шаудера может нарушаться.

В настоящее время известно множество обобщений конической теоремы Красносельского, эффективно работающих в приложениях, однако на практике порой приходится сталкиваться с эффектами, близкими к содержанию конической теоремы, но формально не укладывающимися в ее рамки. Именно к такого рода постановкам приводит задача устойчивого деления на страны двумерной области с априорно распределенным населением.

В классической постановке теорема Брауэра обобщается на многозначные отображения в виде теоремы Какутани. В этой теореме утверждается существование неподвижной точки у точечно-множественного отображения, имеющего замкнутый график и сопоставляющего точкам из X его непустые выпуклые подмножества. В настоящей работе теорема Какутани обобщается на случай отображения $F: X \Rightarrow \mathbb{R}^{n}$ такого, что возможно $F(x) \nsubseteq X$, но удовлетворяются прочие предположения классической теоремы. В этой обобщенной теореме у отображения постулируются граничные свойства, подобные сжимающему или растягивающему требованиям для однозначных отображений.

\footnotetext{
${ }^{1}$ Например, в известном обобщении Шаудера-Тихонова векторное пространство не обязано быть конечномерным, но локально выпуклым.
} 
В процессе публикации настоящей работы рецензент указал на хорошо забытую работу (Тимохов, 1978). Действительно, несмотря на определенные различия в терминологии и формулировках, теорема из (Тимохов, 1978, с. 103, теорема 3.2) фактически содержит результат, аналогичный нашей теореме 6. Эквивалентность этих подходов устанавливается в лемме 2 , представленной в конце разд. 2, где эти подходы обсуждаются. Представленное мною новое и прямое доказательство, в котором вопрос существования неподвижной точки сводится к непосредственному применению теоремы Брауэра, также имеет самостоятельный интерес. Эти разработки изложены в Приложении. B (Marakulin, 2018) теорема Какутани обобщается на случай отображения $F: X \Rightarrow \mathbb{R}^{n}$ такого, что возможно $F(x) \nsubseteq X$, но удовлетворяются прочие предположения классической теоремы. В этой обобщенной теореме у отображения постулируются граничные свойства, подобные сжимающему или растягивающему требованиям для однозначных отображений.

В разд. 1 работы содержится следующий материал: 1) формулируется задача деления области на страны и вводится понятие миграционно стабильного (равновесного) деления на юрисдикции; 2) описывается метод, сводящий ее к поиску неподвижной точки у отображения, заданного на многограннике в конечномерном пространстве, и формулируется теорема 1 о существовании неподвижной точки в соответствующей постановке; 3) формулируется и доказывается теорема 2 о существовании указанного пространственного равновесия. Разд. 2 содержит обобщения теоремы Брауэра (теорема 3), Какутани (теорема 5), их соответствие с теоремой Тимохова (Тимохов, 1978). Заключение и Приложение завершают работу.

\section{1. Задача стабильного деления на страны и ее сведение к неподвижной точке}

Одним из центральных вопросов теории пространственного равновесия является проблема существования иммиграционно состоятельного деления на $n$ стран (число изначально задано) не только на плоскости, но в любом конечномерном пространстве. Это не просто возможное обобщение одномерного случая, но и возможность рассматривать в данном контексте более общие задачи, например деление общества по партийной принадлежности. Это проблема существования равновесия Тибу (Tiebout, 1956) ${ }^{2}$, что весьма важно в контексте многих экономических теорий, таких как теория клубов, финансирование локальных общественных благ, или в политических науках - деление на партии, фракции и т.д. Используемый здесь принцип миграционной состоятельности предполагает, что у жителей отсутствуют выявленные мотивы для изменения юрисдикции, в частности, в данной точке размещения населения имеется равенство издержек у граждан граничных стран. Требуется, чтобы межстрановая граница была пред-

\footnotetext{
2 Общая идея этого равновесия состоит в том, что индивидуумы могут голосовать ногами, т.е. жители страны или муниципалитета способны перемещаться и выбирать то место жительства, которое их больше устраивает. А возможно, и не нужно физически перемещаться, например при формировании фанов футбольных клубов достаточно просто прописаться и затем потреблять все блага и недостатки этого членства.
} 
ставлена непрерывной кривой (поверхностью). В частности, данный принцип стабильности означает, что не существует ненулевой массы населения такой, чтобы членам образующей ее группы было выгодно непрерывное (постепенная трансформация, гомотопия) изменение текущей межстрановой границы. Мы докажем существование надлежащего деления на страны для любой измеримой плотности населения.

Начальное построение аналогично предложенному в (Savvateev et al., 2018): требуется разделить область $\mathcal{A} \subset \mathbb{R}^{l}$ на $n$ стран, $N=\{1, \ldots, n\}$. Распределение населения на $\mathcal{A}$ задано посредством вероятностной меры $\mu(\cdot)$. Для каждой страны $i \in N$ определена функция затрат $c_{i}(\cdot)$, которая может зависеть от массы $\delta_{i} \in[0,1]$ страны и масс других стран, позиции индивида $x \in \mathcal{A}$ и еще от дополнительных параметров $y \in Y$. В частности, в качестве $y$ могут использоваться центры стран (причем потенциально у каждой страны может быть несколько центров - точек размещения локальных общественных благ), а также и другие значимые в странообразовании параметры. Предполагается, что функции затрат непрерывно зависят от $\delta \in \Delta^{(n-1)}$ (стандартный симплекс в $\mathbb{R}^{n}$ ) и $y$, причем $Y$ - область изменения $y$ - выпукла и компактна. Возможность рассмотрения функции затрат столь общего вида отличает данную работу от первоначальной версии (Savvateev et al., 2018), изложенные здесь результаты существенно более общие, охватывают случай переменных центров и т.д.

Предположение Р. Распределение населения на $\mathcal{A}$ описывается абсолютно непрерывной вероятностной мерой $\mu$.

Предположение С. Для каждого $i \in N$ затратъ $c_{i}(\cdot)$ oпределенъь и непрерывны на $\mathcal{A} \times Y\left(\Delta^{(n-1)} \backslash F_{i}\right), \quad$ где $F_{i}=\left\{\delta \in \Delta^{(n-1)} \mid \delta_{i}=0\right\}$, и при любой $\left(x, y, \delta_{i}, \delta_{-i}\right) \rightarrow\left(\bar{x}, \bar{y}, 0, \bar{\delta}_{-i}\right)$, т.е. когда $\bar{\delta}_{i}=0$, имеет место ${ }^{3}$ $c_{i}\left(x, y, \delta_{1}, \ldots, \delta_{n}\right) \rightarrow+\infty$.

Главное отличие предположения $\mathbf{C}$ от предположений, сделанных в работах (Savvateev et al., 2018; Marakulin, 2016), состоит в том, что теперь допускается, чтобы множество потенциально пограничных индивидов имело ненулевую массу. Ранее в дополнение к предположению $\mathbf{C}$ предполагалось, что множество $A_{i j}(y, \delta)=\left\{x \in \mathcal{A} \mid c_{i}(x, y, \delta)=c_{j}(x, y, \delta)\right\}$ имеет нулевую меру Лебега для всех $j \neq i$ и любых $(y, \delta) \in Y \times \Delta^{(n-1) 4}$. Сейчас уже множество $A_{i j}(y, \delta)$ может не быть пренебрежимым. Кроме того, в предположении $\mathbf{P}$ теперь возможно, чтобы $\operatorname{supp}(\mu) \neq \mathcal{A}$, т.е. в области $\mathcal{A}$ допускаются незаселенные участки. Кроме того, необходимо заметить, что теперь одномерный случай также укладывается в общую схему и может быть изучен одновременно с многомерным вариантом ${ }^{5}$.

Основной моделью, представляющей функции, удовлетворяющие предположению $\mathbf{C}$, являются

$$
c_{i}\left(x, y, \delta_{i}, r_{c}\left(S_{i}\right)\right)=g_{i} / \delta_{i}+\rho\left(x, r_{c}\left(S_{i}\right)\right), g_{i}>0, \quad i \in N .
$$

\footnotetext{
${ }^{3}$ Это условие с большим запасом: как показывает анализ леммы 1 , достаточно предполагать .

${ }^{4}$ Отметим также другие отличия предположения $\mathbf{C}$ от предположений, использованных в (Savvateev et al., 2018): наличие $Y$ и зависимость и непрерывность затрат относительно всех переменных.

5 Легко убедиться, что в одномерной постановке множество может не быть пренебрежимо малым для стран интервального вида с центрами в середине отрезка и при затратах вида (1). Поэтому ранее одномерный случай изучался самостоятельно.
} 
Здесь $S_{i} \subset \mathcal{A}$ - это юрисдикция с номером $i$, имеющая массу популяции $\delta_{i}=\mu\left(S_{i}\right), r_{c}\left(S_{i}\right)$ - расположение ее центра (фиксированное или переменное), $\rho(\cdot, \cdot)$ - метрика (возможность находить расстояние до центра от расположения индивидуума, заданного координатами $x \in \mathcal{A})$. Скалярная величина $g_{i}>0$ представляет агрегированный платеж, который требуется для формирования страны $i \in N$ (стоимость правительства) и оплачивается всеми гражданами в равных долях. Таким образом, индивидуальные затраты делятся на два типа: равный для каждого жителя платеж и индивидуализированные затраты, определенные как расстояние от месторасположения жителя до центра страны. Отметим, что предположение $\mathbf{C}$ выполняется и в случае различных центров $A_{i j}(y, \delta)$ представляет возможную границу между странами $i$ и $j$; для евклидовой метрики - это пересечение $\mathcal{A}$ с одной из ветвей гиперболы.

Модель, основанная на функции издержек вида (1), впервые появляется в работе (Alesina, Spolaore, 1997), где излагается общая постановка задачи деления на страны и в одномерной постановке обосновывается применение затрат в функциональном виде (1). В общем случае затраты индивида $c_{i}(\cdot)$ - это своеобразная функция полезности со знаком «минус», которая, вообще говоря, зависит не только от его местонахождения, массы стран и их центров, но и от переменного параметра $y \in Y$. В многомерный вектор $y$ может быть включено много параметров, в том числе уровень благосостояния во всех возможных странах и обеспеченность населения локальными общественными благами по месту жительства (в этой обеспеченности и состоит функция государства на модельном уровне, именно поэтому так важны столицы, где исторически больше общественных благ и выше уровень благосостояния). В многозначном случае (описанное ниже деление на юрисдикции может быть неоднозначно) вместо затрат (1) в качестве базисной мы рассматриваем модель вида

$$
c_{i}\left(x, y, \delta_{i}, r_{c}\left(S_{i}\right)\right)=\max \left\{t_{i}, g_{i} / \delta_{i}\right\}+\min \left\{d_{i}, \rho\left(x, r_{c}\left(S_{i}\right)\right)\right\}, i \in N .
$$

Здесь величина $t_{i}>0$ интерпретируется как минимальный налог на каждого гражданина страны $i, g_{i}>0$ - платеж на содержание правительства, $d_{i}>0$ - расстояние, начиная с которого влияние удаленности от центра становится безразличным. Представляется, что затраты вида (2) реалистичнее предыдущих.

Теперь мы дадим формальное определение равновесного (иммиграционно стабильного) деления на юрисдикции.

Определение 1 . Пусть $\mathcal{A} \subset \mathbb{R}^{l}$ - область конечномерного пространства. Совокупность замкнутых подмножеств $S_{i} \subset \mathcal{A}, i \in N$, называется миграчионно-состоятельным равновесием, если удовлетворяет требованиям:

i) $\cup_{N} S_{i}=\mathcal{A}, \delta_{i}=\mu\left(S_{i}\right)>0, i \in N, \sum_{N} \delta_{i}=1$ и $\exists y \in Y$ такой, что

ii) $\forall i \neq j, c_{i}\left(x, y, \delta_{1}, \ldots, \delta_{n}\right)=c_{j}\left(x, y, \delta_{1}, \ldots, \delta_{n}\right) \forall x \in S_{i} \cap S_{j}$,

iii) $\forall i \in N \quad S_{i} \supseteq\left\{x \in \mathcal{A} \mid c_{i}\left(x, y, \delta_{1}, \ldots, \delta_{n}\right)<c_{j}\left(x, y, \delta_{1}, \ldots, \delta_{n}\right) \forall j \in N: j \neq i\right\}$. 
Предъявленные здесь требования означают:

i) подмножества $S_{i} \subset \mathcal{A}$ образуют нетривиальное разбиение области на юрисдикции такие, что каждый индивид приписан к некоторой юрисдикции и число тех, которые имеют гражданство нескольких стран, пренебрежимо мало;

ii) пограничные жители нескольких стран имеют одинаковые издержки;

iii) каждая юрисдикция включает в себя всех тех жителей, которым наиболее выгодно быть ее членами.

На самом деле несложно заключить, что требование іi) следует из i) и іiі) и приводится с целью прояснить смысл концепции в целом. Отметим, что при затратах типа (2) возможно $\mu\left(A_{i j}(y, \delta)\right)>0$, и в таком случае может быть (бесконечно) много вариантов равновесного деления на юрисдикции (подробности ниже в контексте доказательства и построения точечно-множественного отображения).

Идея доказательства обобщенной теоремы существования равновесного деления на юрисдикции состоит в том, чтобы набору $\left(\delta_{1}, \ldots, \delta_{n}, y\right)$ из номинальных параметров поставить в соответствие аналогичный набор реальных параметров, рассчитанный для иммиграционного стабильного деления, заданного номинальными переменными. Однако сопоставляемый набор теперь может быть не единственным, но представимым в $\Delta^{(n-1)}$ в виде выпуклого замкнутого подмножества потенциально допустимых вариантов. Тем самым определяется точечно-множественное отображение, чья нетривиальная неподвижная точка удовлетворяет требованиям деления на юрисдикции. Опишем эту конструкцию более подробно.

$\mathrm{B}$ пространстве $\mathbb{R}^{n}$ рассмотрим стандартный симплекс $\Delta^{(n-1)}$ и отображения, заданные по следующим формулам.

Пусть $s(x, \delta, y)=\min _{j \in N}\left\{c_{j}(x, \delta, y)\right\},(\delta, y) \in \Delta^{(n-1)} \times Y$. Определим далее границы, в рамках которых может изменяться масса страны. Пусть $\theta_{i}=\mu\left(S_{i}(\delta, y)\right), \theta_{-i}=\mu\left(S_{-i}(\delta, y)\right)$, где по определению

$$
\begin{gathered}
S_{i}(\delta, y)=\left\{x \in \mathcal{A} \mid c_{i}(x, \delta, y)=s(x, \delta, y) \& c_{j}(x, \delta, y)>s(x, \delta, y) \quad \forall j \neq i\right\}, \\
S_{-i}(\delta, y)=\left\{x \in \mathcal{A} \mid c_{i}(x, \delta, y)>s(x, \delta, y)\right\} .
\end{gathered}
$$

Тогда массы $\mu_{i}$ стран могут изменяться в пределах

$$
\theta_{i} \leq \mu_{i} \leq 1-\theta_{-i}, \quad i \in N, \sum_{i \in N} \mu_{i}=1 .
$$

Здесь $\mu_{i}-\theta_{i}-$ масса населения страны $i$ из числа тех граждан, кому безразлично присоединяться к стране $i$ или к другой стране; $\left(1-\theta_{i}-\theta_{-i}\right)$ - общий объем этой массы, $\theta_{-i}-$ масса жителей, которые определенно не будут гражданами страны $i$.

Искомое распределение массы населения между странами можно осуществить и несколько иным, но эквивалентным способом. Разобьем область $\mathcal{A}$ на подмножества: для $T \subseteq N, T \neq \varnothing$ определим 


$$
S_{T}(\delta, y)=\left\{x \in \mathcal{A} \mid c_{i}(x, \delta, y)=s(x, \delta, y) \quad \forall i \in T \& c_{j}(x, \delta, y)>s(x, \delta, y) \quad \forall j \notin T\right\}
$$

и $S_{\varnothing}(\delta, y)=\varnothing$. Положим $\mathrm{v}_{T}=\mu\left(S_{T}\right)$ и определим $d^{T}=\left(d_{i}^{T}\right)_{i \in T}$ из условия $\sum_{i \in T} d_{i}^{T}=v_{T}, d_{i}^{T} \geq 0, i \in T$. Для заданной конфигурации $(\delta, y) \in \Delta^{(n-1)} \times Y$ найдем массы стран $\left(\mu_{i}\right)_{i \in N}$ по формуле $\mu_{i}=\sum_{T \subseteq N: i \in T} d_{i}^{T}, i \in N$.

Таким образом, конфигурации $(\delta, y)$ ставится в соответствие множество $\mathcal{F}(\delta, y)$ возможных вариантов распределения массы популяции такое, что если $T \subseteq N$ - это группа стран, $v_{T}=\mu\left(S_{T}\right)>0$ - масса граждан, для которых безразлично, с какой из юрисдикций $i \in T$ они аффилированы, то $v_{T}$ распределяется среди этих стран любым возможным способом. Более того, так как $S_{T}(\delta, y) \cap S_{R}(\delta, y)=\varnothing$ при $S \neq R$ и $\cup_{T \subseteq N} S_{T}(\delta, y)=\mathcal{A}$, то

$$
\sum_{T \subseteq N} \mathrm{v}_{T}=\mu(\mathcal{A})=1 \Rightarrow \sum_{i \in N} \mu_{i}=\sum_{i \in N}\left(\sum_{T \subseteq N: i \in T} d_{i}^{T}\right)=\sum_{T \subseteq N} \sum_{i \in T} d_{i}^{T}=\sum_{T \subseteq N} \mathrm{v}_{T}=1 .
$$

Кроме того, в соответствии с конструкцией предыдущего абзаца $\theta_{i}=d_{i}^{\{i\}}$ и, следовательно, $\mu_{i}-\theta_{i}=\sum\left\{d_{i}^{T}|T \subseteq N: i \in T| T \mid, \geq 2\right\}$.

В итоге, на $\Delta^{(n-1)} \times Y$ указанным образом определено точечномножественное отображение $\mathcal{F}:(\delta, y) \rightarrow \mathcal{F}(\delta, y) \subseteq \Delta^{(n-1)}$, которое имеет выпуклые замкнутые значения.

Имеется также некоторое непрерывное отображение ${ }^{6}$ $\mathcal{M}: \Delta^{(n-1)} \times Y \rightarrow Y$. Содержательно это отображение аккумулирует в себе все непротиворечивые требования, которые на модельном уровне могут предъявляться к миграционно состоятельному делению на юрисдикции. Например, для заданной текущей конфигурации юрисдикций так могут находиться центры стран как центры тяжести или в целом это размещение центров локальных общественных благ и т.д. В терминах работы (Savvateev et al., 2018) это могут быть параметры, определяющие характеристики потребительских сообществ, провайдеров общественных благ, размещения производственных мощностей, размеры платежей разного рода и т.д. В результате мы приходим к итоговому отображению $[\mathcal{F} \times \mathcal{M}](\delta, y)=\mathcal{F}(\delta, y) \times \mathcal{M}(\delta, y), \quad(\delta, y) \in \Delta^{(n-1)} \times Y$.

Далее нам достаточно найти нетривиальную неподвижную точку $\bar{\delta}=\left(\bar{\delta}_{1}, \ldots, \bar{\delta}_{n}\right) \in \Delta^{(n-1)}, \bar{y} \in Y$ этого отображения, т.е.

$$
\bar{\delta} \in \mathcal{F}(\bar{\delta}, \bar{y}), \quad \bar{y}=\mathcal{M}(\bar{\delta}, \bar{y}): \quad \bar{\delta}=\left(\bar{\delta}_{1}, \ldots, \bar{\delta}_{n}\right)>>0 .
$$

Этот факт будет установлен с помощью доказанной в Приложении теоремы 5 и ее следствия - теоремы 1. С этой целью нам потребуется доказать лемму, но сначала мы напомним понятие «отображения Какутани».

Определение 2. Пусть Х, Y замкнутые подмножества некоторых линейных пространств. Точечно-множественное отображение $F: X \Rightarrow Y$ называется отображением Какутани, если для каждого $x \in X$ значение $F(x) \neq \varnothing$, выпукло и график $G r F=\{(x, y) \in X \times Y \mid y \in F(x)\}$ замкнут.

\footnotetext{
${ }^{6}$ При необходимости тоже можно считать многозначным отображением Какутани.
} 
Лемма 1. Пусть предположения $\mathbf{P}$ и $\mathbf{C}$ выполнены. Тогда для некоторого $0<\varepsilon<1 / n$ :

i) $\mathcal{F}(\cdot)$ является отображением Какутани на $\Delta_{\varepsilon}^{(n-1)} \times Y$;

ii) $\forall y \in Y \mathcal{F}(\cdot, y)$ отображает $\varepsilon$-подсимплекс $\Delta_{\varepsilon}^{(n-1)}=\left\{\delta \in \mathbb{R}^{n} \mid \sum \delta_{i}=\right.$ $\left.=1, \delta_{i} \geq \varepsilon \forall i \in N\right\} \quad$ так, что грани $\Delta_{\varepsilon}^{(n-1)}$ отображаются в соответ ствуюшие грани исходного симплекса, т.е. $\left[\delta=\left(\delta_{i}, \delta_{-i}\right) \in \Delta_{\varepsilon}^{(n-1)} \& \delta_{i}=\right.$ $=\varepsilon] \Rightarrow \mu_{i}(\delta, y)=0, \mathcal{F}(\delta, y)=\left(\mu_{1}(\delta, y), \ldots, \mu_{n}(\delta, y)\right)$.

Д о к а з а т е л ь с т в о. С целью установить пункт і) покажем, что отображение $\mathcal{F}$ имеет замкнутый график. Рассмотрим семейство $\left(\delta_{\xi}, y_{\xi}\right) \rightarrow(\bar{\delta}, \bar{y}), \xi \in \Xi$, точек из $\Delta^{(n-1)} \times Y$ и отвечающее ему семейство $\left(\mu_{i}^{\xi}\right)_{i \in N} \in \mathcal{F}\left(\delta_{\xi}, y_{\xi}\right)$ такое, что $\left(\mu_{i}^{\xi}\right)_{i \in N} \rightarrow\left(\bar{\mu}_{i}\right)_{i \in N}$. Требуется доказать, что $\left(\bar{\mu}_{i}\right)_{i \in N} \in \mathcal{F}(\bar{\delta}, \bar{y})$.

Для действительного $\alpha>0$ и произвольного $(\delta, y) \in \Delta^{(n-1)} \times Y$ определим множества

$$
\begin{aligned}
S_{i}^{\alpha}(\delta, y)= & \left\{x \in \mathcal{A} \mid c_{i}(x, \delta, y)<\min _{j \neq i, j \in N} c_{j}(x, \delta, y)-\alpha\right\}, \\
& \mu_{i}^{\alpha}(\delta, y)=\mu\left(S_{i}^{\alpha}(\delta, y)\right), i \in N .
\end{aligned}
$$

Ясно, что $S_{i}^{\alpha}(\delta, y) \subset S_{i}^{\beta}(\delta, y) \subset S_{i}(\delta, y)$ при $\alpha>\beta>0$ и в силу предположений $\mathbf{P}$ и $\mathbf{C}$ величины $\mu\left(S_{i}^{\alpha}(\delta, y)\right) \rightarrow \mu\left(S_{i}(\delta, y)\right)$ при $\alpha \downarrow 0$. Отметим, что из предположения $\mathbf{C}$ для каждого действительного $\varepsilon>0$ затраты равномерно непрерывны на $\mathcal{A} \times Y \times \Delta_{\varepsilon}^{(n-1)}$. Это влечет, что для любой фиксированной пары $(\delta, y)$, для каждого $\alpha>0$ существует действительное $\gamma>0$ такое, что для каждого $\delta^{\prime} \in \Delta_{\varepsilon}^{(n-1)},|| \delta^{\prime}-\delta||<\gamma$ и каждого $y^{\prime} \in Y$, ||$y^{\prime}-y||<\gamma$ имеем:

$$
\begin{gathered}
\forall i \in N, c_{-i}(x, \delta, y)=\min _{j \neq i, j \in N} c_{j}(x, \delta, y) \\
\forall x \in \mathcal{A} \quad\left|c_{i}(x, \delta, y)-c_{i}\left(x, \delta^{\prime}, y^{\prime}\right)\right|<\frac{\alpha}{3} \&\left|c_{-i}(x, \delta, y)-c_{-i}\left(x, \delta^{\prime}, y^{\prime}\right)\right|<\frac{\alpha}{3} \Rightarrow \\
\Rightarrow \exists \bar{\xi} \in \Xi: \forall \xi \geq \bar{\xi}, \forall x \in \mathcal{A}\left|c_{i}(x, \bar{\delta}, \bar{y})-c_{i}\left(x, \delta_{\xi}, y_{\xi}\right)\right|<\frac{\alpha}{3} \& \\
\&\left|c_{-i}(x, \bar{\delta}, \bar{y})-c_{-i}\left(x, \delta_{\xi}, y_{\xi}\right)\right|<\frac{\alpha}{3} \Rightarrow S_{i}^{\alpha}(\bar{\delta}, \bar{y}) \subset S_{i}^{\frac{\alpha}{3}}\left(\delta_{\xi}, y_{\xi}\right) \Rightarrow \\
\Rightarrow \mu\left(S_{i}^{\alpha}(\bar{\delta}, \bar{y})\right) \leq \mu\left(S_{i}^{\frac{\alpha}{3}}\left(\delta_{\xi}, y_{\xi}\right)\right) \leq \mu\left(S_{i}\left(\delta_{\xi}, y_{\xi}\right)\right)=\theta_{i}\left(\delta_{\xi}, y_{\xi}\right) \leq \mu_{i}^{\xi} .
\end{gathered}
$$

Переходя в этом неравенстве к пределу по $\xi \in \Xi$, заключаем $\mu\left(S_{i}^{\alpha}(\bar{\delta}, \bar{y})\right) \leq \bar{\mu}_{i}$, откуда в силу

$$
\lim _{\alpha \downarrow+0} \mu\left(S_{i}^{\alpha}(\bar{\delta}, \bar{y})\right)=\mu\left(S_{i}(\bar{\delta}, \bar{y})\right)=\theta_{i}(\bar{\delta}, \bar{y}) \Rightarrow \theta_{i}(\bar{\delta}, \bar{y}) \leq \bar{\mu}_{i} \quad \forall i \in N .
$$

Таким образом, установлено первое из неравенств (3). Второе требуемое в (3) неравенство доказывается подобным образом, но теперь уже с использованием множеств

$$
S_{-i}^{\alpha}(\delta, y)=\left\{x \in \mathcal{A} \mid c_{i}(x, \delta, y)>\min _{j \neq i, j \in N} c_{j}(x, \delta, y)+\alpha\right\} .
$$

Поскольку равенство в ограничениях $(3)$ для $\left(\bar{\mu}_{i}\right)_{N}$ достигается путем обычного предельного перехода, считаем $\left(\bar{\mu}_{i}\right)_{N} \in \mathcal{F}(\bar{\delta}, \bar{y})$ доказанным. 
Чтобы установить ii), фиксируем $i$, положим $\Delta_{\varepsilon i}^{(n-1)}=$. $=\Delta_{\varepsilon}^{(n-1)} \cap\left\{x \in \mathbb{R}^{n} \mid x_{i}=\varepsilon\right\}$ и найдем такие $\delta^{\varepsilon} \in \Delta_{\varepsilon i}^{(n-1)}, \quad\left(x^{\varepsilon}, y^{\varepsilon}\right) \in \mathcal{A} \times Y$, что $c_{i}\left(x^{\varepsilon}, y^{\varepsilon}, \delta^{\varepsilon}\right)=\inf _{(x, y, \delta) \in \mathcal{A} \times Y \times \Delta_{\varepsilon i}^{(n-1)}} c_{i}(x, y, \delta)$. Без ограничения общности можно считать, что $\left(x^{\varepsilon}, y^{\varepsilon}, \delta^{\varepsilon}\right) \rightarrow(\bar{x}, \bar{y}, \bar{\delta})$ при $\varepsilon \rightarrow 0$. Имеем $\delta_{i}^{\varepsilon}=\varepsilon \rightarrow \bar{\delta}_{i}=0$ и в силу предположения $\mathbf{C}$ заключаем $c_{i}\left(x^{\varepsilon}, y^{\varepsilon}, \delta^{\varepsilon}\right) \rightarrow+\infty$. В то же время для каждого $j$ такого, что $\bar{\delta}_{j}>0$ затраты $c_{j}\left(x, y, \delta^{\varepsilon}\right)$ ограничены сверху для всех $(x, y) \in \mathcal{A} \times Y$ и малых $\varepsilon>0$. Таким образом, для всех достаточно малых $\varepsilon>0$,

$$
\begin{gathered}
c_{i}\left(x, y, \delta^{\varepsilon}\right)>\inf _{j \neq i, j \in N} c_{j}\left(x, y, \delta^{\varepsilon}\right) \quad \forall(x, y) \in \mathcal{A} \times Y \Rightarrow S_{-i}\left(y, \delta^{\varepsilon}\right)=\mathcal{A} \Rightarrow \\
\theta_{-i}\left(y, \delta^{\varepsilon}\right)=1 \Rightarrow \mu_{i}=0 \forall\left(\mu_{j}\right)_{j \in N} \in \mathcal{F}\left(y, \delta^{\varepsilon}\right),
\end{gathered}
$$

что и требовалось доказать.

С учетом леммы 1 нижеследующая теорема позволяет утверждать, что описанное отображение $\mathcal{F} \times \mathcal{M}$ имеет неподвижную точку B $\Delta_{\varepsilon}^{(n-1)} \times Y$.

Напомним, что в линейном пространстве $L$ аффинная оболочка $\operatorname{aff}(X)$ множества $X \subset L$ это $\operatorname{aff}(X)=\left\{\sum_{\Xi} \lambda_{\xi} x_{\xi}\left|x_{\xi} \in X, \lambda_{\xi} \in \mathbb{R} \forall \xi \in \Xi,\right| \Xi \mid<\right.$ $\left.<\infty, \sum_{\Xi} \lambda_{\xi}=1\right\}$. Аффинная оболочка может быть также описана как $\operatorname{aff}(X)=x+\mathcal{L}(X-x)$, где $\mathcal{L}(X-x)-$ линейнал оболочка $(X-x)$, и $x \in X$ выбран произвольно.

Пусть $M \subset \mathbb{R}^{n}-$ выпуклый ограниченный многогранник и $\operatorname{aff}(M)-$ его аффинная оболочка. Пусть $d \in \operatorname{ri} M-$ некоторая точка из относительной внутренности многогранника $M, F_{t}, t=1, \ldots, m,-$ его грани максимальной нетривиальной размерности (на единицу меньше размерности $M)$. С каждой гранью свяжем конус $K_{t} \subset \operatorname{aff}(M)$ с вершиной в точке $d: K_{t}=\left\{d+\lambda(\kappa-d) \mid \kappa \in F_{t}, \quad \lambda \geq 0\right\} \Rightarrow \operatorname{aff}(M)=\underset{t=1, \ldots, m}{\cup} K_{t}$.

Теорема 1. Пусть $\mathcal{F}: M \Rightarrow \operatorname{aff}(M)$ - отображение Какутани, опре-

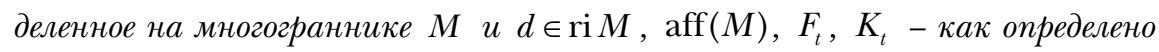
выше. Пусть выполняется одно из условий:

i) cжатие (compressive form): $\mathcal{F}\left(F_{t}\right) \subset M \quad \forall t=1, \ldots, m$;

ii) растяжение (expansive form): $\mathcal{F}\left(F_{t}\right) \subset K_{t} \backslash$ ri $M \quad \forall t=1, \ldots, m$.

Тогда $\mathcal{F}$ имеет неподвижную точку на $M$.

Результат теоремы 1 в варианте расширяющего условия представлен на рис. 1. Здесь $f$ непрерывно отображает меньший симплекс $\Delta_{\varepsilon}^{(n-1)}$ на его расширение $\Delta^{(n-1)}$ и подчиняется условию ii). Доказательство тео-

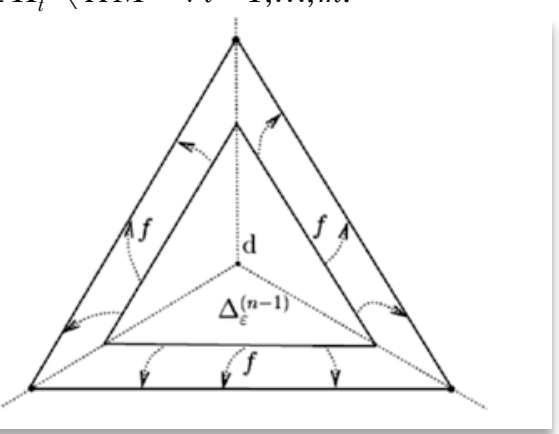

Рис. 1

Исходный и вложенный симплекс $\Delta^{(n-1)}$ и отображение $f(\cdot)$ 
ремы 1 следует из теоремы 5 и характеризации граничных точек многогранника в терминах опорных гиперплоскостей (опущено). Одним из основных результатов работы является следующая теорема.

Теорема 2. Пусть $\mathcal{A}$ - компактное подмножество конечномерного линейного пространства и $\mu$ - мера на $\mathcal{A}$. Если выполненъ предположения $\mathbf{P}, \mathbf{C}$, то область $\mathcal{A}$ можно разделить на любое заданное число иммиграчионно состоятельных юрисдикиий ненулевого оббема. Это деление также можно осуществить так, чтобы выполнялисъ заданные непрерывные непротиворечивые требования.

Д о к а з а т е л с т в о. Непосредственно следует из описанных выше построений, ибо в условиях леммы 1 применима теорема 1.

Итак, доказано, что произвольная ограниченная область любого многомерного пространства может быть разделена на страны, даже если существует ненулевая масса людей, для которых безразлична принадлежность к данной стране или некоторой другой. Однако теорема 2 представляет только размеры стран и, возможно, некоторые дополнительные параметры (столицы и др.). При этом границы стран непосредственно не указываются. Возникает вопрос, как их нужно определить. Ответ заключается в том, что существует множество возможных границ между странами, но они должны подчиняться определенным ограничениям.

Предположим, что для страны $i \in N$ ее границы определяют область $S_{i} \subset \mathcal{A}$. Тогда должно быть $\mu\left(S_{i}\right)=\delta_{i}$ и $S_{i}=S_{i}(\delta, y) \supseteq$ $\supseteq\left\{x \in \mathcal{A} \mid c_{i}(x, y, \delta)<c_{j}(x, y, \delta) \forall j \neq i\right\}$, где множество в правой части включения представляет всех жителей, которые безусловно хотят быть гражданами страны $i$. Таким образом, любая межстрановая конфигурация, в которой страны подчиняются этому требованию, может рассматриваться как возможный вариант деления на страны. По построению это устойчивое равновесное разбиение. Рис. 2 иллюстрирует данную ситуацию. Область $\mathcal{A}$, подлежащая делению, очерчена. Страны $A$,

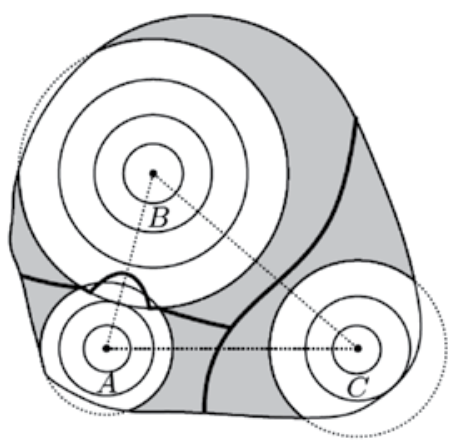

Puc. 2

Деление области на страны $A, B, C$
$B, C$ разделены границей (полужирная линия). У стран заданы центры, издержки типа (2), расстояние евклидовое. Затемненная область - это зона, жителям которой безразлично, в какой стране они имеют гражданство.

Замечание. Общая идея представленной конструкции была заимствована из (Savvateev et al., 2018). Эти построения были модифицированы и приспособлены к случаю странообразования с затратами, которые могут 
зависеть также от дополнительных параметров $y \in Y$. Данное обобщение имеет большое значение, поскольку позволяет исследовать случай переменных центров стран, в том числе в теоретически интересном случае, когда центры стран выбираются как барицентры (центр масс) постранового расселения жителей. Построение, учитывающее многозначность отображения $\mathcal{F}$, вполне оригинально. Таким образом, предложенный нами метод, основанный на применении обобщенной теоремы Какутани, является достаточно гибким и позволяет получать новые значимые результаты экономической теории.

\section{2. Обобщенные теоремы о неподвижных точках}

В данном разделе описываются некоторые обобщения классических теорем о неподвижной точке Брауэра-Какутани, развитие и применение которых оказалось востребованным в рамках проблемы существования пространственного равновесия.

Начнем обсуждение с обобщения теоремы Брауэра.

Пусть далее $\partial X-$ это граница множества $X$ в его аффинной оболочке, т.е. $\partial X=X \backslash$ ri $X$, где $\operatorname{ri} X$ - относительная внутренность замкнутого выпуклого множества $X \subset L$.

Теорема 3. Пусть $X \subset \mathbb{R}^{n}-$ выпуклый компакт и $f: X \rightarrow \operatorname{aff}(X)-$ непрерывное отображение со значениями в аффинной оболочке $X$. Предположим, ито $f$ удовлетворяет одному из условий:

i) сжатие (compressive form)

$$
\forall x \in \partial X, \forall h \in \mathbb{R}^{n}[\langle h, x\rangle \geq\langle h, X\rangle \Rightarrow\langle h, f(x)\rangle \leq\langle h, x\rangle] ;
$$

ii) растяжение (ехpansive form)

$$
\forall x \in \partial X, \forall h \in \mathbb{R}^{n}[\langle h, x\rangle \geq\langle h, X\rangle \Rightarrow\langle h, f(x)\rangle \geq\langle h, x\rangle] .
$$

Тогда $f$ имеет неподвижную точку в $X$.

Отметим, что при $\operatorname{aff}(X) \supsetneqq \operatorname{aff}(f(X))$ условия теоремы в части требований (4), (5) в изложенном виде могут оказаться трудновыполнимыми. Однако в таком случае их можно существенно ослабить. Если $X \cap \operatorname{aff}(f(X))$ имеет непустую внутренность в $\operatorname{aff}(f(X))$, то (4), (5) можно требовать только для точек из $\partial X \cap \operatorname{aff} f(f(X))$. Чтобы убедиться в этом, в изложенном ниже доказательстве нужно рассмотреть снижение отображения $f$ на $X \cap \operatorname{aff}(f(X))$ и установить существование неподвижной точки на этом множестве. Проверка непосредственная.

Анализ доказательства показывает, что утверждение теоремы 3 можно обобщить на случай декартова произведения отображений, первое из которых удовлетворяет условию теоремы 3, а второе - условиям теоремы Брауэра или условиям, приводимым к ней, например, выполнены условия і) или іi).

Теорему 3 можно переформулировать в несколько ином компактном виде. С этой целью напомним, что с выпуклым замкнутым подмножеством $X$ линейного пространства $L$ и $x^{*} \in X$ в выпуклом ана- 
лизе принято ассоциировать конусы двух видов: 1) нормальный конус $\mathfrak{N}_{X}\left(x^{*}\right)=\left\{h \in L^{*} \mid\left\langle h, x^{*}-X\right\rangle \geq 0\right\}$, где $L^{*}-$ двойственное пространство к $L ; 2)$ касательный конус -

$$
\mathfrak{T}_{X}\left(x^{*}\right)=\left\{y \in L \mid\left\langle h, x^{*}-y\right\rangle \geq 0 \quad \forall h \in \mathfrak{N}_{X}\left(x^{*}\right)\right\}=\operatorname{cl}\left(\bigcup_{\lambda>0} \lambda\left(X-x^{*}\right)\right) .
$$

Теперь условие (4) можно переписать в терминах касательного конуса в виде: $\forall x^{*} \in \partial X\left(f\left(x^{*}\right)-x^{*}\right) \in \mathfrak{T}_{X}\left(x^{*}\right)$. Если учесть, что aff $X=\mathfrak{T}_{X}\left(x^{*}\right)+x^{*}$ при $x^{*} \in \operatorname{ri} X$ и $\mathfrak{T}_{X}\left(x^{*}\right)+x^{*} \subset \operatorname{aff} X$ при $x^{*} \in \partial X$, в итоге получим $\forall x^{*} \in X\left(f\left(x^{*}\right)-x^{*}\right) \in \mathfrak{T}_{X}\left(x^{*}\right)$. Подобного рода заключения имеют место и для условия (5). Тем самым приходим к новой формулировке теоремы 3.

Теорема 4. Пусть $X \subset \mathbb{R}^{n}$ - непустой выпуклый компакт $и$ $f: X \rightarrow \mathbb{R}^{n}$ - непрерывное отображение. Предположим, ито $f$ удовлетворяет одному из условий:

i) cжатие (compressive form): $\left(f\left(x^{*}\right)-x^{*}\right) \in \mathfrak{T}_{X}\left(x^{*}\right) \forall x^{*} \in X$;

ii) растяжение (expansive form): $\left(x^{*}-f\left(x^{*}\right)\right) \in \mathfrak{T}_{X}\left(x^{*}\right) \forall x^{*} \in X$.

Тогда а имеет неподвижную точку в $X$.

Классическая теорема Какутани обобщает теорему Брауэра на многозначные отображения (точечно-множественные). Здесь допускается, чтобы значения отображения были множествами, но непременно непустыми выпуклыми компактами - своеобразная обобщенная точка. Требование непрерывности отображения заменяется на замкнутость графика или на эквивалентную ему полунепрерывность сверху. Однако в классической версии теоремы Какутани, так же, как и в теореме Брауэра, предполагается, что значения отображения должны быть подмножествами его (выпуклой и компактной) области определения. Однако подобно теореме Брауэра теорему Какутани о существовании неподвижной точки у точечно-множественного отображения можно обобщить на случай, когда отображение может действовать и за пределы области определения.

Как и ранее, будем предполагать, что $X \subset \mathbb{R}^{n}-$ выпуклый комnaкm и, без ограничения общности, int $X \neq \varnothing$. Однако теперь мы рассмотрим точечно-множественное отображение (соответствие) $F: X \Rightarrow \mathbb{R}^{n}$. Напомним следующее классическое определение полунепрерывности сверху.

Определение 3. Точечно-множественное отображение $F: X \Rightarrow Y \subset \mathbb{R}^{n}$ называется полунепрерывным сверху в точке $x \in X$, если $F(x) \neq \varnothing$ и для каждого открытого $U \supset F(x)$ найдется окрестность $V_{x}$ точки $x$ такая, что $F(z) \subset U \quad \forall z \in V_{x}$.

Отображение $F: X \Rightarrow Y \subset \mathbb{R}^{n} \quad$ называется полунепрерывным сверху, если оно полунепрерывно сверху в каждой точке $X$.

Подчеркнем, что в нашем случае возможно $F(x) \nsubseteq X, x \in X$. Известно, что если $X \times Y$ компакт, отображение $F: X \Rightarrow Y \subset \mathbb{R}^{n}$ имеет 
замкнутый график и $F(x) \neq \varnothing \quad \forall x \in X$, то оно полунепрерывно сверху. Таким образом, если $X \times Y$ компакт, отображение Какутани полунепрерывно сверху.

Рассмотрим далее дополнительные граничные требования на $F(\cdot)$. Для $x \in \partial X$ может требоваться -

$$
\text { сжатие: } \exists y \in F(x) \quad \forall h \in \mathbb{R}^{n}[\langle h, x\rangle \geq\langle h, X\rangle \Rightarrow\langle h, y\rangle \leq\langle h, x\rangle]
$$

или

растяжение: $\exists y \in F(x) \quad \forall h \in \mathbb{R}^{n}[\langle h, x\rangle \geq\langle h, X\rangle \Rightarrow\langle h, y\rangle \geq\langle h, x\rangle]$.

Отображения Какутани, для которых требование (6) выполнено в каждой граничной точке $X$, мы называем сжимюжими. Аналогично, если во всех граничных точках отображение удовлетворяет (7), его мы называем растягиваюиим.

Теорема 5. Пусть $X \subset \mathbb{R}^{n}$ - непустой выпуклый компакт, $Y \subset \mathbb{R}^{n}-$ компактно и $F: X \Rightarrow Y \subset \mathrm{a} f(X)$ точечно-множественное отображение Какутани, где $\operatorname{aff}(X)$ - аффинная оболочка $X$. Тогда если отображение $F(\cdot)$ сжимающее или, альтернативно, растягивающее, то $F$ имеет неподвижную точку на X.

Подобно случаю однозначных отображений для многозначного случая последняя теорема может быть переформулирована в терминах касательных конусов.

Теорема 6. Пусть $X \subset \mathbb{R}^{n}$ - непустой выпуклый компакт u $F: X \Rightarrow Y \subset \mathbb{R}^{n}$ - точечно-множественное отображение Какутани. Предположим, ито $F$ удовлетворяет одному из условий:

i) сжатия- $\left(F\left(x^{*}\right)-x^{*}\right) \cap \mathfrak{T}_{X}\left(x^{*}\right) \neq \varnothing \quad \forall x^{*} \in X$;

ii) растяжения $-\left(x^{*}-F\left(x^{*}\right)\right) \cap \mathfrak{T}_{X}\left(x^{*}\right) \neq \varnothing \quad \forall x^{*} \in X$.

Тогда $F$ имеет неподвижную точку в $X$.

Оригинальное доказательство этой и предыдущих теорем содержится в Приложении.

В литературе по прикладным методам анализа имеется работа (Тимохов, 1978) ${ }^{7}$, результаты которой частично покрывают содержание теоремы 6. А именно в работе утверждается (в рафинированной форме), что точечно-множественное отображение Какутани $F: X \Rightarrow \mathbb{R}^{l}$, определенное на выпуклом телесном (непустая внутренность) компакте $X \subset \mathbb{R}^{l}$, имеет неподвижную точку в $X$, если $F(X)$ ограничено и выполнено одно из условий:

$$
\begin{aligned}
& \forall x \in \partial X, \quad \forall y \in F(x) \quad \exists h \in \mathbb{R}^{l}, \quad h \neq 0:\langle h, X\rangle \leq\langle h, x\rangle, \quad\langle h, y\rangle \leq\langle h, x\rangle, \\
& \forall x \in \partial X, \forall y \in F(x) \quad \exists h \in \mathbb{R}^{l}, \quad h \neq 0:\langle h, X\rangle \leq\langle h, x\rangle, \quad\langle h, y\rangle \geq\langle h, x\rangle .
\end{aligned}
$$
или

Первое требование подобно нашему сжатию, а второе - растяжению. Однако есть и отличия. Например, тот факт, что условие (9) влечет растяжение в нашем смысле, не является очевидным и требует доказательства. Аналогичное утверждение справедливо и для условия (8). В следующей лемме рассматривается посылка (9).

\footnotetext{
${ }^{7}$ Я благодарен анонимному рецензенту за указание на данную работу.
} 


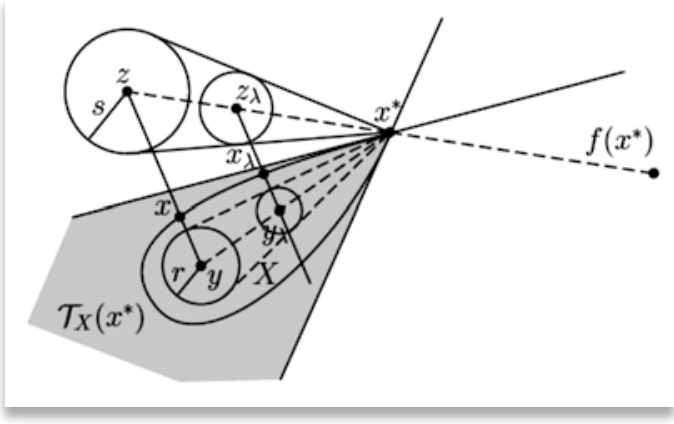

Pис. 3

Геометрия доказательства леммы 2
Лемма 2. Пусть $X \subset \mathbb{R}^{l}-$ выпуклый компакт, $\operatorname{int} X \neq \varnothing \quad u$ $F: X \Rightarrow \mathbb{R}^{l}$-отображение Какутани такое, что $F(X)$ ограничено. Тогда если выполнено (9), то $F$ удовлетворлет условию (ii) теоремъ 6 и, следовательно, имеет неподвижную точку.

Д ок а 3 а т ел ь с т в о. Для простоты рассмотрим случай однозначного отображения $f: X \rightarrow \mathbb{R}^{l} \quad$ (несложно распространяется на общий случай).

Предположим противное и найдем точку $x^{*} \in \partial X$, удовлетворяющую $\left(x^{*}-f\left(x^{*}\right)\right) \notin \mathfrak{T}_{X}\left(x^{*}\right)$. Здесь $\mathfrak{T}_{X}\left(x^{*}\right)$ - ограниченный, замкнутый, выпуклый касательный конус. Последующие построения иллюстрирует рис. 3 , на котором касательный конус представлен затененной областью.

Рассмотрим произвольную точку $y \in \operatorname{int} X$ и обозначим через $B_{r}=\left\{y \in \mathbb{R}^{l} \mid\|y\|<r\right\}$ открытый шар радиуса $r>0$ (с центром в нуле). Найдутся $r>0, s>0$ такие, что

$$
\left(B_{r}+y\right) \subset X, \quad\left(B_{s}+x^{*}-f\left(x^{*}\right)\right) \cap \mathfrak{T}_{X}\left(x^{*}\right)=\varnothing .
$$

Из построения очевидно, что если $z=x^{*}+\left[x^{*}-f\left(x^{*}\right)\right]$ и точка $x \in \mathbb{R}^{l}$ находится на линейном отрезке $[z, y]$, соединяющем $у$ и $z$, причем ||$x-y|| \geq r,|| x-z|| \geq s$, то

$$
\begin{gathered}
\forall h \in \mathbb{R}^{l}:\langle h, x\rangle>\left\langle h, y+B_{r}\right\rangle \Rightarrow\langle h, x\rangle\left\langle\langle h, \zeta\rangle \quad \forall \zeta \in \mathbb{R}^{l}:\right. \\
|| \zeta-z||<s r /(|| z-y||-s) .
\end{gathered}
$$

Последнее можно переписать в виде

$$
\langle h, x\rangle<\left\langle h, z+B_{\varepsilon}\right\rangle \quad \forall 0<\varepsilon \leq s r /(|| z-y||-s) .
$$

По построению (10) выполнено для любого функционала $h, o n o p$ ного $^{8}$ в граничной точке множества $X$ из отрезка $[z, y]$.

Далее рассмотрим линейный отрезок $\left[y, x^{*}\right]=\left\{\lambda y+(1-\lambda) x^{*} \mid\right.$ $0 \leq \lambda \leq 1\}$, текущей точке которого $y_{\lambda}=\lambda y+(1-\lambda) x^{*}$ поставим в соответствие две точки: $z_{\lambda}=\lambda z+(1-\lambda) x^{*}$ и точку $x_{\lambda}$, полученную как пересечение $\partial X$ с отрезком $\left[y_{\lambda}, z_{\lambda}\right]$. Любой опорный функционал в точке $x_{\lambda}$ обладает теми же свойствами (10), однако теперь уже в отношении величин $\lambda s>0, \lambda r>0$ и ||$z_{\lambda}-y_{\lambda}||=\lambda|| z-y||$. Зафиксируем $\varepsilon>0$, удовлетворяющий (10), и выберем $\bar{\lambda}>0$ так, чтобы $f\left(x_{\lambda}\right) \in f\left(x^{*}\right)+B_{\varepsilon}$ $\forall \lambda \in(0, \bar{\lambda})$, что возможно в силу непрерывности $f$. Теперь, при $0<\lambda<\bar{\lambda}$ для каждого опорного в точке $x_{\lambda}$ функционала $h \in \mathbb{R}^{l}$ имеем

$$
\left\langle h, x_{\lambda}\right\rangle \leq\left\langle h, z_{\lambda}+\lambda B_{\varepsilon}\right\rangle, \quad 0<\varepsilon \leq \frac{\lambda s r}{\lambda(|| z-y||-s)}=\frac{s r}{|| z-y||-s} .
$$

\footnotetext{
${ }^{8}$ Я благодарен анонимному рецензенту за указание на данную работу.
} 
Отсюда, в частности, следует, что это свойство справедливо и для выбранного фиксированного $\varepsilon>0$. Значит, с учетом опорности $h$ можно заключить:

$$
\left\langle h, x^{*}\right\rangle \leq\left\langle h, x_{\lambda}\right\rangle\left\langle\left\langle h, z_{\lambda}+\lambda\left(f\left(x^{*}\right)-f\left(x_{\lambda}\right)\right)\right\rangle .\right.
$$

Подставляя значение $z_{\lambda}$ и опуская средний член неравенства, получаем

$$
\begin{gathered}
\left\langle h, x^{*}\right\rangle\left\langle\left\langle h, \lambda\left(x^{*}+\left[x^{*}-f\left(x^{*}\right)\right]\right)+(1-\lambda) x^{*}+\lambda\left(f\left(x^{*}\right)-f\left(x_{\lambda}\right)\right)\right\rangle \Rightarrow\right. \\
\left.0<\left\langle h, \lambda\left[x^{*}-f\left(x^{*}\right)\right]+\lambda\left(f\left(x^{*}\right)-f\left(x_{\lambda}\right)\right)\right\rangle \Rightarrow\left\langle h, x^{*}\right\rangle>\left\langle h, f\left(x_{\lambda}\right)\right)\right\rangle .
\end{gathered}
$$

Так как $\left\langle h, x_{\lambda}\right\rangle \geq\left\langle h, x^{*}\right\rangle$, тем самым приходим к $\left.\left.\left\langle h, x_{\lambda}\right\rangle\right\rangle\left\langle h, f\left(x_{\lambda}\right)\right)\right\rangle$, что справедливо для всех функционалов $h \in \mathbb{R}^{l}$, опорных к $X$ в точке $x_{\lambda}$. Это противоречит исходному предположению (9).

Чтобы распространить этот результат на многозначный случай, достаточно использовать рассуждения, подобные изложенным выше, с целью доказать $\left(x^{*}-F\left(x^{*}\right)\right) \cap \mathfrak{T}_{X}\left(x^{*}\right) \neq \varnothing \quad \forall x^{*} \in \partial X$, и далее применить теорему 6.

Несложно убедиться, что результат, аналогичный лемме 2, но рассмотренный относительно предпосылки (8) и сжимающего условия теоремы 6 , также имеет место. Таким образом, любая из предпосылок (8) и (9) достаточна, для того чтобы ограниченное отображение Какутани, определенное на выпуклом компакте, имело в нем неподвижную точку. При этом утверждение теоремы 4 очевидно следует из теоремы Тимохова, а значит, как было выше доказано, справедлива и теорема 6. Таким образом, эти теоремы эквивалентны и любая из них может служить обоснованием теоремы 1.

\section{Заключение}

В работе изучается задача деления на страны (сообщества) компактной области в конечномерном пространстве с априорно заданной плотностью распределения населения. Число стран изначально определено. Требуется найти такое межстрановое деление, чтобы у жителей не было стимула менять свою юрисдикцию, в частности, граждане разных стран, живущие в граничных точках, имеют одинаковые издержки. Показано, что вопрос существования стабильного в этом смысле межстранового деления можно свести к нахождению неподвижной точки у некоторого точечно-множественного отображения. При этом оказалось, что известные теоремы о неподвижной точке (Брауэра, Какутани) неприменимы, ибо построенное отображение может действовать за пределы области определения, хотя и удовлетворяет некоторому граничному растягивающему свойству. Это вызвало разработку и доказательство обобщений классических теорем, позволяющих установить требуемое деление на страны. Развитые методы и результаты могут применяться во многих разделах экономической теории, в том числе теории клубов, локальных общественных благ, в политических науках при партийном формировании и т.д. 


\section{ПРИЛОЖЕНИЕ}

Доказательства обобщенных теорем о неподвижной точке

Будем предполагать, что $X \subset \mathbb{R}^{n}=L-$ выпуклый компакт, $f: X \rightarrow \mathbb{R}^{n}$ и, без ограничения общности, int $X \neq \varnothing$. Определим отображение

$$
\alpha(x)=\sup \left\{\alpha \in \mathbb{R}_{+} \mid x+\alpha(x-f(x)) \in X\right\}, \quad x \in X .
$$

Если $x \not f(x)$ и луч $L_{-}(x)=\left\{x+\mu(x-f(x)) \mid \mu \in \mathbb{R}_{+}\right\}$пересекается с int $X$, т.е. при $L_{-}(x) \cap \operatorname{int} X \neq \varnothing$, значение $\alpha(x)$ определено корректно. Аналогично, при $L_{+}(x) \cap \operatorname{int} X \neq \varnothing$, где $L_{+}(x)=\left\{x+\mu(f(x)-x) \mid \mu \in \mathbb{R}_{+}\right\}$, можно рассматривать отображение

$$
\beta(x)=\sup \left\{\beta \in \mathbb{R}_{+} \mid x+\beta(f(x)-x) \in X\right\}, \quad x \in X .
$$

Лемма 3. Пусть $f: X \rightarrow \mathbb{R}^{n}$ непрерывно в точке $x \in X$ u $x \neq f(x)$. Тогда если $L_{-}(x) \cap \operatorname{int} X \neq \varnothing$, то отображение $\alpha(\cdot)$ определено в окрестности $x$, непрерывно в этой точке $u \alpha(x)>0$. Если $L_{+}(x) \cap \operatorname{int} X \neq \varnothing$, то $\beta(\cdot)$ определено, непрерьвно в окрестности х и $\beta(x)>0$.

Д о к а з а т е л ь с т в о. Рассмотрим первое заключение (доказательство второго аналогично). В условиях леммы достаточно доказать непрерывность $\alpha(\cdot)$ в точке $x$. Пусть $x_{\xi} \rightarrow x, \xi \in \Xi$, установим $\alpha\left(x_{\xi}\right) \rightarrow \alpha(x)$. Пусть $\bar{\alpha}-$ любая предельная точка семейства $\alpha_{\xi}=\alpha\left(x_{\xi}\right)$, $\xi \in \Xi$. Без ограничения общности можно считать, что $\alpha_{\xi} \rightarrow \bar{\alpha}$. Достаточно показать, что $\bar{\alpha}=\alpha(x)$. Сделаем это.

По построению все точки $y_{\xi}=x_{\xi}+\alpha_{\xi}\left(x_{\xi}-f\left(x_{\xi}\right)\right)$ являются граничными в $X$, т.е. $y_{\xi} \in \partial X, \quad \xi \in \Xi$. В то же время $y_{\xi}=x_{\xi}+\alpha_{\xi}\left(x_{\xi}-f\left(x_{\xi}\right)\right) \rightarrow x+\bar{\alpha}(x-f(x))=y$. Так как $\partial X$ замкнуто, то $y \in \partial X$, однако по предположению, $\exists z^{\prime} \in L_{-}(x) \cap \operatorname{int} X$. Тогда имеем $z^{\prime}=x+\alpha^{\prime}(x-f(x))$, где $0<\alpha^{\prime}<\alpha(x)$, и значит, внутренняя точка $z^{\prime}$ находится на линейном отрезке $\operatorname{co}\{x, z\}, z=x+\alpha(x)(x-f(x))$, соединяющем две разные точки из $X$. Известно, что для выпуклых множеств это возможно только если все точки вида $\lambda x+(1-\lambda) z, 0<\lambda<1$ являются внутренними в $X$. Однако $y \in c o\{x, z\}$ и является граничной точкой. Значит, $y=z$ или $y=x$. Покажем, что $y=x$ невозможно.

Чтобы убедиться в этом, возьмем произвольный $z^{\prime}=x+\alpha^{\prime}(x-f(x)) \in \operatorname{int} X, \quad 0<\alpha^{\prime}<\alpha(x)$ и найдем окрестности нуля $V, W$ такие, что $(V+x) \cap(W+f(x))=\varnothing$ и

$$
f(V+x) \subset(W+f(x)) \& U=\left(1+\alpha^{\prime}\right) V-\alpha^{\prime} W+z^{\prime} \subset \operatorname{int} X .
$$

Возьмем произвольный $v \in V, x^{\prime}=x+v$, положим $f\left(x^{\prime}\right)=f(x)+w$, $w \in W$ и рассмотрим $z^{\prime \prime}=x^{\prime}+\alpha^{\prime}\left(x^{\prime}-f\left(x^{\prime}\right)\right)$. Подставляя значения, имеем $z^{\prime \prime}=x+v+\alpha^{\prime}(x+v-f(x)-w)=\left[x+\alpha^{\prime}(x-f(x))\right]+\left(1+\alpha^{\prime}\right) v-\alpha^{\prime} w$, откуда $z^{\prime \prime} \in U$. Следовательно, если $x_{\xi} \in V+x$ и $f\left(x_{\xi}\right) \in W+f(x)$, то $y_{\xi}=x_{\xi}+\alpha^{\prime}\left(x_{\xi}-f\left(x_{\xi}\right)\right) \in U$. Но тогда из определения (11) заклю- 
чаем: $\alpha\left(x_{\xi}\right)>\alpha^{\prime}$ для всех достаточно больших $\xi \in \Xi$ и, значит, $\bar{\alpha}>0$ и $x+\bar{\alpha}(x-f(x))=y \neq x$.

Доказано, что $y=x+\alpha(x)(x-f(x))=z$, откуда следует $\bar{\alpha}=\alpha(x)$. Таким образом, $\alpha(x)$ является единственной предельной точкой семейства $\alpha\left(x_{\xi}\right), \xi \in \Xi$ и, значит, $\alpha\left(x_{\xi}\right) \underset{\Xi}{\rightarrow} \alpha(x)$.

Далее нас будут интересовать альтернативные условия, обеспечивающие непрерывность отображений $\alpha(\cdot), \beta(\cdot)$ и полезные в дальнейшем анализе. С этой целью рассмотрим лемму.

Лемма 4. Пусть $X \subset L$ выпукло, замкнуто, int $X \neq \varnothing, x \in \partial X u$ $y \in L$ удовлетворяет $\forall h \in L^{*}, h \neq 0[\langle h, X\rangle \leq\langle h, x\rangle \Rightarrow\langle h, x\rangle\langle\langle h, y\rangle]$. Тогда в $X$ найдется окрестность $V_{x}$ точки $x$ такая, что

$$
\left.\{z+\mu(z-y)) \mid \mu \in \mathbb{R}_{+}\right\} \cap \operatorname{int} X \neq \varnothing \quad \forall z \in V_{x} .
$$

Д о к а з а т е л ь с т в о. Покажем, что $L_{-}(x, y) \cap \operatorname{int} X \neq \varnothing$, где $\left.L_{-}(x, y)=\{x+\mu(x-y)) \mid \mu \in \mathbb{R}_{+}\right\}$. Предполагая противное, применим стандартную теорему отделимости к множествам, участвующим в последнем пересечении, и найдем линейный $h \neq 0$ такой, что $\langle h, X\rangle \leq\left\langle h, L_{-}(x, y)\right\rangle$. Так как $x \in L_{-}(x, y)$, то $\langle h, x\rangle \geq\langle h, X\rangle$ и $\langle h, y\rangle \leq\langle h, x\rangle$ (неравенство $\langle h, y\rangle\rangle\langle h, x\rangle$ влечет $\inf \left\langle h, L_{-}(x, y)\right\rangle=-\infty$, что противоречит отделимости). Таким образом, найдены точка и функционал, нарушающие предположение леммы, что невозможно.

Рассмотрим общий случай. Пусть $x_{\mu} \in L_{-}(x, y) \cap \operatorname{int} X$. Тогда найдется открытая выпуклая окрестность $U$ точки $x_{\mu}$ такая, что $U \subset$ int $X$. Далее образуем множество (конический отрезок) $W_{x}=\cup_{\lambda \in[0,1)}[(1-\lambda) U+\lambda y]$ и рассмотрим его пересечение с $X$. По построению $W_{x}$ открыто, $x \in W_{x}$ и, очевидно, $\left.\{z+\mu(z-y)) \mid \mu \in \mathbb{R}_{+}\right\} \cap \operatorname{int} X \neq \varnothing$ $\forall z \in V_{x}=W_{x} \cap X$, что и требовалось доказать.

Рассмотрим два условия на отображение $f: X \rightarrow \mathbb{R}^{n}:$ для каждого граничного $x \in \partial X$ и каждого линейного $h \neq 0$, опорного к $X$ в точке $x$, т.е. если $\langle h, X\rangle \leq\langle h, x\rangle$, имеет место

$$
\langle h, f(x)\rangle<\langle h, x\rangle
$$

или

$$
\langle h, f(x)\rangle>\langle h, x\rangle \text {. }
$$

Первое требование будем называть сильно сжимающим свойством, а второе - сильно расиирлющиим (рис. 4).

Лемма 5. Пусть $f: X \rightarrow \mathbb{R}^{n}-$ непрерывное отображение. Тогда если $f$ обладает сильно сжимающим свойством, то $\beta(\cdot)$ определено, непрерывно на $X$ и $\beta(x)>0$ для всех $x \in X$ таких, ито $f(x) \neq x$; если выполнено

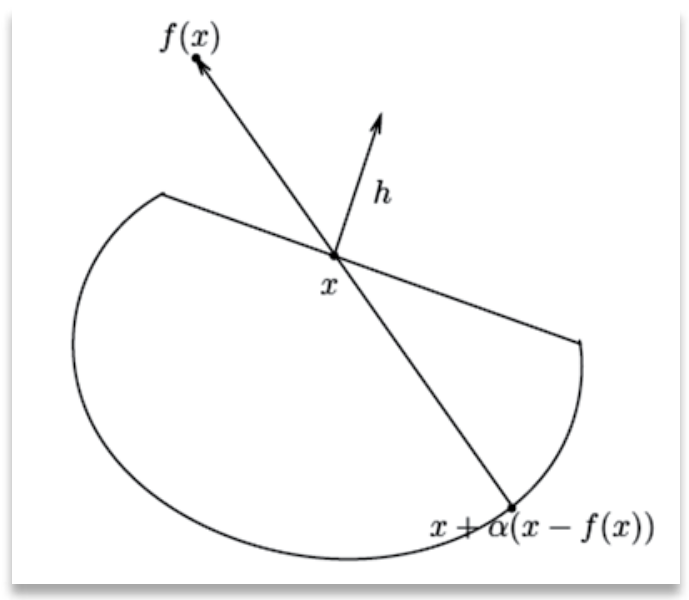

Рис. 4

Расширяющее свойство (14) 
сильно расширяющее, то $\alpha(\cdot)$ определено, непрерывно $u \alpha(x)>0$ для $x \in X$ : $f(x) \neq x$.

Д ок а за те ль с т о. Достаточно рассмотреть первое заключение. Применяя лемму 4 , в условиях леммы 5 заключаем: $\forall x \in X$, если $x \neq f(x)$, то $L_{+}(x) \cap \operatorname{int} X \neq \varnothing$, где луч $L_{+}(x)$ был определен выше (см. (11), (12)). Теперь мы находимся в условиях леммы 3, утверждение которой дает искомый результат.

Теорема 7. Пусть $X \subset \mathbb{R}^{n}-$ выпуклый компакт $u$ int $X \neq \varnothing$. Тогда каждое непрерывное отображение $f: X \rightarrow \mathbb{R}^{n}$, обладающее сильно сжимающим или расширяюшим свойством, имеет неподвижную точку в $X$.

Д о к а з а т е л ь с т в о. Предположим, заключение теоремы ложно и $f(x) \neq x \forall x \in X$. Пусть, например, выполнено расширяющее свойство. Определим отображение

$$
g(x)=x+\alpha(x)(x-f(x)), x \in X,
$$

где функция $\alpha(\cdot)$ была определена выше в (11). По построению $g(x) \in X$ $\forall x \in X$ и в силу леммы $5 g(\cdot)$ непрерывно на $X$. Следовательно, в силу классической теоремы Брауэра $g(\cdot)$ имеет неподвижную точку $\bar{x} \in X$. Имеем $\bar{x}=\bar{x}+\alpha(\bar{x})(\bar{x}-f(\bar{x})) \Rightarrow \alpha(\bar{x})(\bar{x}-f(\bar{x}))=0 \Rightarrow \alpha(\bar{x})=0$. Однако в силу леммы 3 должно быть $\alpha(\bar{x})>0$ - противоречие. Следовательно, $f(\cdot)$ имеет неподвижную точку на $X$.

\section{Доказательство теоремы 3}

Предположим, без ограничения общности, что int $X \neq \varnothing$ и $0 \in \operatorname{int} X$. Пусть $f$ удовлетворяет (4). Для произвольного действительного $1>\varepsilon>0$ рассмотрим вспомогательное отображение $v(x)=(1-\varepsilon) f(x)$. Покажем, что $v(x)$ удовлетворяет (13). Действительно, так как $0 \in \operatorname{int} X$, то для $h \neq 0,\langle h, x\rangle \geq\langle h, X\rangle$, имеем $\langle h, x\rangle>0$. При $\langle h, f(x)\rangle>0$ в силу (4) имеем $\langle h, f(x)\rangle \leq\langle h, x\rangle$, откуда $\langle h,(1-\varepsilon) f(x)\rangle\langle\langle h, x\rangle$. Если $\langle h, f(x)\rangle \leq 0$, то условие $\langle h,(1-\varepsilon) f(x)\rangle\langle\langle h, x\rangle$ выполнено автоматически. Следовательно, в силу теоремы 7 для каждого $\varepsilon>0$ отображение $v(\cdot)$ имеет неподвижную точку $x_{\varepsilon} \in X: x_{\varepsilon}=v\left(x_{\varepsilon}\right)$.

В силу компактности $X$ можно считать, что $x_{\varepsilon} \rightarrow \bar{x}$ при $\varepsilon \rightarrow 0$. Переходя в равенстве $(1-\varepsilon) f\left(x_{\varepsilon}\right)=x_{\varepsilon}$ к пределу по $\varepsilon \rightarrow 0$ находим $\bar{x}=f(\bar{x})$. Таким образом, для $f(\cdot)$ установлено существование неподвижной точки на $X$.

В случае когда $f$ удовлетворяет (5), рассмотрим отображение $w(x)=(1+\varepsilon) f(x)$. При $h \neq 0,\langle h, x\rangle \geq\langle h, X\rangle$ имеем $\langle h, f(x)\rangle \geq\langle h, x\rangle>0$, откуда следует $\langle h,(1+\varepsilon) f(x)\rangle\rangle\langle h, x\rangle$. Это означает, что $w(x)$ удовлетворяет условию (14). Таким образом, если выполнено (5), то в силу теоремы 7 отображение $w(\cdot)$ имеет неподвижную точку $x_{\varepsilon}$. Далее рассуждаем аналогично предыдущему случаю - конструируем сходящееся при $\varepsilon \rightarrow 0$ семейство неподвижных точек и, переходя к пределу в равенстве 
$(1+\varepsilon) f\left(x_{\varepsilon}\right)=x_{\varepsilon}$, устанавливаем существование неподвижной точки у $f(\cdot)$.

\section{Доказательство теоремы 5}

Без ограничения общности предполагаем, что $0 \in \operatorname{int} X \neq \varnothing$. Для действительного $\lambda$ рассмотрим отображение $G(x)=(1+\lambda) F(x)$. Пусть, например, для $x \in \partial X$ отображение $F$ удовлетворяет граничному требованию (7). Фиксируем $\lambda>0$. Для линейного $h \neq 0$ при $\langle h, x\rangle \geq\langle h, X\rangle$ условие $0 \in \operatorname{int} X$ влечет $\langle h, x\rangle>0$. Поэтому для отображения $G$ предположение (7) будет выполнено в строгой форме неравенства и, применяя лемму 5, будем иметь $\exists y \in G(x): \forall h \in \mathbb{R}^{n}, h \neq 0[\langle h, x\rangle \geq\langle h, X\rangle \Rightarrow$ $\Rightarrow\langle h, y\rangle>\langle h, x\rangle] \Rightarrow \quad\left\{x+\mu(x-y) \in \mathbb{R}^{n}: \mu \in \mathbb{R}_{+}\right\} \cap$ int $X \neq \varnothing$. Более того, последнее свойство будет выполнено не только в точке $x$, но и в точках из некоторой ее окрестности. В итоге, $\forall x \in \partial X$ существует открытая окрестность $V_{x}$ этой точки (шар):

$$
\left.\exists y \in G(x): \forall h \in \mathbb{R}^{n}, h \neq 0[\langle h, x\rangle \geq\langle h, X\rangle \Rightarrow\langle h, y\rangle\rangle\langle h, z\rangle \forall z \in V_{x}\right] .
$$

Построим надлежащую точечно-точечную аппроксимацию отображения $G$. Для этого выберем любой действительный $\varepsilon>0$ и рассмотрим открытое покрытие $\partial X$ множествами вида $V_{x}^{\varepsilon}=V_{x} \cap\left\{z \in \mathbb{R}^{n}|||z-x| \mid<\varepsilon\right\}$. Можно считать, что это открытые шары радиуса $0<\varepsilon_{x} \leq \varepsilon$. В силу компактности $\partial X$ можно выбрать конечное подпокрытие, ассоциированное с семейством точек (центры шаров) $x_{i} \in \partial X, i=1, \ldots, k$, таких, что $\partial X \subset \bigcup_{i=1}^{k} V_{x_{i}}^{\varepsilon}=W$.

Далее рассмотрим покрытие компактного множества $X \backslash W$ открытыми шарами $U_{x}$ с центром в точке $x$ радиуса $0<\varepsilon_{x} \leq \varepsilon$ и такими, что $U_{x} \cap \partial X=\varnothing$. Найдем конечное подпокрытие $U_{x_{j}}^{\varepsilon}$, при $j=k+1, \ldots, k+l$. Имеем

$$
X \backslash W \subset \bigcup_{j=1}^{l} U_{x_{k+j}}^{\varepsilon} \& \partial X \bigcap\left(\bigcup_{j=1}^{l} U_{x_{k+j}}^{\varepsilon}\right)=\varnothing .
$$

Для элементов первого и второго покрытия определим функции:

$$
\begin{gathered}
z \in \mathbb{R}^{n}, \\
\alpha_{i}(z)=\max \left\{0, \varepsilon_{i}-|| z-x_{i}||\right\}, \quad i=1, \ldots, k ; \\
\beta_{j}(z)=\max \left\{0, \varepsilon_{k+j}-|| z-x_{k+j}||\right\}, \quad j=1, \ldots, l
\end{gathered}
$$

и итоговое отображение $g^{\varepsilon}(\cdot): X \rightarrow \mathbb{R}^{l}$ по формуле

$$
g^{\varepsilon}(x)=\left\{\sum_{i=1}^{k} \alpha_{i}(x) y_{i}+\sum_{j=1}^{l} \beta_{j}(x) y_{k+j}\right\} /\left\{\sum_{i=1}^{k} \alpha_{i}(x)+\sum_{i=1}^{l} \beta_{j}(x)\right\} .
$$

Здесь значение $y_{i} \in G\left(x_{i}\right), i=1, \ldots, k$, выбрано так, чтобы выполнялось требование (15), а точки $y_{k+j} \in G\left(x_{k+j}\right), j=1, \ldots, l$ - произвольным образом. Эта функция определена корректно, так как каждая точка $X$ принадлежит хотя бы одному элементу покрытия и, значит, стоящая в знаменателе величина отлична от нуля. Очевидно, что это непрерывная 
функция. Важным ее свойством является тот факт, что $\sum_{i=1}^{l} \beta_{j}(x)=0$ в граничных точках $x \in \partial X$, где ее значение задается формулой

$$
g^{\varepsilon}(x)=\sum_{i=1}^{k} \alpha_{i}(x) y_{i} / \sum_{i=1}^{k} \alpha_{i}(x), \quad x \in \partial X
$$

Более того, по определению $V_{x_{i}}^{\varepsilon}$ из $\alpha_{i}(x)>0$ следует $x \in V_{x_{i}}^{\varepsilon}$, что означает $\left\{x+\mu\left(x-y_{i}\right) \in \mathbb{R}^{n}: \mu \in \mathbb{R}_{+}\right\} \cap \operatorname{int} X \neq \varnothing$. Выпуклая комбинация точек $y_{i}$, обладающих этим свойством, также удовлетворяет этому требованию. Отсюда следует, что $\left\{x+\mu\left(x-g^{\varepsilon}(x)\right) \in \mathbb{R}^{n}: \mu \in \mathbb{R}_{+}\right\} \cap \operatorname{int} X \neq \varnothing$. Но это означает, что $g^{\varepsilon}(x)$ удовлетворяет (14), т.е. выполнено $\left.\forall x \in \partial X \forall h \in \mathbb{R}^{n}, h \neq 0\left[\langle h, x\rangle \geq\langle h, X\rangle \Rightarrow\left\langle h, g^{\varepsilon}(x)\right\rangle\right\rangle\langle h, x\rangle\right]$. Таким образом, в силу теоремы 7 отображение $g^{\varepsilon}(\cdot)$ имеет неподвижную точку в $X$.

На следующем этапе мы осуществляем предельный переход. Действительно, к данному моменту доказано, что $\forall \varepsilon>0$ $\exists x^{\varepsilon} \in X: x^{\varepsilon}=g^{\varepsilon}\left(x^{\varepsilon}\right)$. Однако $X$ компакт, поэтому без ограничения общности можно считать, что $x^{\varepsilon} \rightarrow \bar{x}, \bar{x} \in X$ при $\varepsilon \rightarrow 0$. Покажем, что $\bar{x} \in G(\bar{x})$. С этой целью воспользуемся полунепрерывностью сверху точечно-множественного отображения $G(x)=(1+\lambda) F(x)$. По определению для каждого $\gamma>0$ существует $\eta>0$ такая, что ||$x-\bar{x}||<\eta$ $\Rightarrow|| y-G(\bar{x})||<\gamma \forall y \in G(x)$. Возьмем $0<\varepsilon<\eta / 2$ и выберем $\bar{\varepsilon}>0$ так, чтобы ||$x^{\varepsilon}-\bar{x}||<\eta / 2$ при $0<\varepsilon<\bar{\varepsilon}$. Теперь, так как $\alpha_{i}\left(x^{\varepsilon}\right)>0$ возможно, только если ||$x_{i}^{\varepsilon}-x^{\varepsilon}||<\varepsilon$, то (неравенство треугольника) ||$x_{i}^{\varepsilon}-\bar{x}||<\eta$. В то же время, по построению $y_{i}^{\varepsilon} \in G\left(x_{i}^{\varepsilon}\right)$ и, так как $G\left(x_{i}^{\varepsilon}\right) \subset G(\bar{x})+B^{\gamma}$, где $B^{\gamma}$ открытый шар радиуса $\gamma>0$ с центром в нуле. Таким образом,

$$
\forall i\left[\alpha_{i}\left(x^{\varepsilon}\right)>0 \Rightarrow y_{i}^{\varepsilon} \in G(\bar{x})+B^{\gamma}\right] \Rightarrow x^{\varepsilon}=g^{\varepsilon}\left(x^{\varepsilon}\right) \in \operatorname{co}\left(G(\bar{x})+B^{\gamma}\right)=G(\bar{x})+B^{\gamma} .
$$

В итоге имеем $x^{\varepsilon} \rightarrow \bar{x} \in \bigcap_{\gamma>0}\left(G(\bar{x})+B^{\gamma}\right)=G(\bar{x})$, т.е. $\bar{x} \in X-$ неподвижная точка $G(\cdot)$.

На завершающем этапе нам потребуется еще один предельный переход. Для каждого $\lambda>0$ существует $x_{\lambda} \in X$ такой, что $x_{\lambda} \in(1+\lambda) F\left(x_{\lambda}\right)$. Это можно переписать в виде

$$
\forall \lambda>0 \exists x_{\lambda} \in X: \frac{x_{\lambda}}{1+\lambda} \in F\left(x_{\lambda}\right) \Leftarrow\left(x_{\lambda}, \frac{x_{\lambda}}{1+\lambda}\right) \in G r F .
$$

Однако по предположению $G r F$ замкнутое подмножество компакта $X \times Y$. Следовательно, любая предельная точка семейства $\left(x_{\lambda}, \frac{x_{\lambda}}{1+\lambda}\right)_{\lambda>0}$
при $\lambda \rightarrow 0$ должна принадлежать графику $F$, т.е.

$$
\exists \bar{x} \in X:\left(x_{\lambda}, \frac{x_{\lambda}}{1+\lambda}\right) \underset{\lambda \downarrow 0}{\rightarrow}(\bar{x}, \bar{x}) \in G r F \Rightarrow \exists \bar{x} \in X: \bar{x} \in F(\bar{x}),
$$

что и требовалось доказать. 


\section{ЛИТЕРАТУРА}

Красносельский М.А. (1960). Неподвижные точки операторов, сжимающих или растягивающих конус / / Доклады Академии наук. Т. 135. № 3. С. 527-530.

Маракулин В.М. (2017). Теория пространственного равновесия: существование иммиграционно состоятельного деления на страны в одномерном пространстве // Сибирский журнал чистой и прикладной математики. T. 17 (4). C. $64-78$.

Тимохов А.В. (1978). Некоторые теоремы о неподвижной точке / / Методы функиионального анализа в математической экономике. Сборник «Проблемы советской экономики». М.: Наука. С. 98-110.

Alesina A., Spolaore E. (1997). On the Number and Size of Nations / / Quarterly Journal of Economics. Vol. 113. P. 1027-1056.

Dugundji J., Granas A. (1982). Fixed Point Theory. Monographie Matematyczne. Warsaw: PWN - Polish Scientific Publishers.

Kwong M.K. (2008). On Krasnoselskii's Cone Fixed Point Theorem // Fixed Point Theory and Applications. Vol. 2008. P. 18. DOI: 10.1155/2008/164537.

Marakulin V.M. (2016). On the Existence of Immigration Proof Partition into Countries in Multidimensional Space. In: Kochetov Yu. et al. (eds) "DOOR-2016. LNCS (Lecture Notes in Computer Sciences)”. Vol. 9869. P. 494-508. Heidelberg: Springer. DOI: 10.1007/978-3-319-44914-2 39.

Marakulin V.M. (2017). Spatial Equilibrium on the Plane and an Arbitrary Population Distribution. In: Evtushenko Yu.G. et al. (eds) "Proceedings of the OPTIMA-2017 Conference”. Petrovac, Montenegro. 02-06 October 2017. P. 378-385.

Marakulin V.M. (2018). Generalized Kakutani Theorem and Spatial Equilibrium Theory. Mimeo.

Musatov M., Savvateev A., Weber S. (2016). Gale-Nikaido-Debreu and MilgromShannon: Communal Interactions with Endogenous Community Structures / / Journal of Economic Theory. Vol. 166. P. 282-303.

Savvateev A., Sorokin C., Weber S. (2018). Multidimensional Free-Mobility Equilibrium: Tiebout Revisited. Mimeo.

Tiebout C.M. (1956). A Pure Theory of Local Expenditures // The Journal of Political Economy. Vol. 64. P. 416-424.

Поступила в редакиию 27.02.20192.

\section{REFERENCES (with English translation or transliteration)}

Alesina A., Spolaore E. (1997). On the Number and Size of Nations. Quarterly Journal of Economics, 113, 1027-1056.

Dugundji J., Granas A. (1982). Fixed Point Theory. Monographie Matematyczne. Warsaw: PWN - Polish Scientific Publishers.

Krasnosel'skii M.A. (1960). Fixed Points of Cone-Compressing or Cone-Extending Operators. Proceedings of the USSR Academy of Sciences, 135, 3, 527-530 (in Russian).

Kwong M.K. (2008). On Krasnoselskii's Cone Fixed Point Theorem. Fixed Point Theory and Applications, 2008, 18. DOI: 10.1155/2008/164537. 
Marakulin V.M. (2016). On the Existence of Immigration Proof Partition into Countries in Multidimensional Space. In: Kochetov Yu. et al. (eds) "DOOR 2016. LNCS (Lecture Notes in Computer Sciences)”, 9869, 494-508. Heidelberg: Springer. DOI: 10.1007/978-3-319-44914-2 39.

Marakulin V.M. (2017). Spatial Equilibrium on the Plane and an Arbitrary Population Distribution. In: Evtushenko Yu.G. et al. (eds) "Proceedings of the OPTIMA-2017 Conference”. Petrovac, Montenegro. 02-06 October 2017, 378-385.

Marakulin V.M. (2017). A Theory of Spatial Equilibrium: The Existence of Migration Proof Country Partition in an Uni-Dimensional World. Siberian Journal of Pure and Applied Mathematics, 17 (4), 64-78 (in Russian).

Marakulin V.M. (2018). Generalized Kakutani Theorem and Spatial Equilibrium Theory. Mimeo.

Musatov M., Savvateev A., Weber S. (2016). Gale-Nikaido-Debreu and MilgromShannon: Communal Interactions with Endogenous Community Structures. Journal of Economic Theory, 166, 282-303.

Savvateev A., Sorokin C., Weber S. (2018). Multidimensional Free-Mobility Equilibrium: Tiebout Revisited. Mimeo.

Tiebout C.M. (1956). A Pure Theory of Local Expenditures. The Journal of Political Economy, 64, 416-424.

Timokhov A.V. (1978). Some Fixed-Point Theorems. Methods of Functional Analysis in Mathematical Economics: "Problems of the Soviet Economy". Moscow: Nauka, $98-110$.

Received 27.02.2019

\section{V.M. Marakulin}

Novosibirsk State University; Sobolev Institute of Mathematics, Russian Academy of Sciences, Novosibirsk, Russia

\section{On the Existence of Spatial Equilibrium and Generalized Fixed Point Theorems}

Abstract. The problem of immigration-proof division into countries of a finite-dimensional area is studied. This is a kind of Tiebout equilibrium, in which the principle of migration consistency assumes that the border residents do not have incentives for the change of jurisdiction, i.e., at the point of their location costs of citizens for border countries are equal. It is assumed that a measurable population density is given and it is required that the cross-country boundary be represented by a continuous surface (curve). The proof of the existence of required division is based on the reducing of the problem to the searching of a fixed point. However, the conditions of classical theorems on fixed points of Brouwer-Kakutani or the "conical" Krasnoselskii's theorem are violated. This led us to the development of their generalization to the case of a mapping, possibly acting outside the domain. It is proved that a continuous mapping defined on a convex compact has a fixed point if it satisfies one of two boundary conditions: "compression" or "expansion". This result also is extended to point-to-set "Kakutani maps": applying it, we prove the existence of an immigration-consistent division into countries of any compact finite-dimensional area in very general setting.

Keywords: Brouwer-Kakutani fixed points theorems, compression and expansion conditions, country formation, migration-consistent (stable) partition.

JEL Classification: D70, H20, H73.

DOI: 10.31737/2221-2264-2019-42-2-1 